\title{
Utilisation de la résistance génétique à la colibacillose K88 dans les schémas d'amélioration génétique du porc
}

\author{
L Ollivier, X Renjifo \\ Institut National de la Recherche Agronomique, Station de génétique \\ quantitative et appliquée, 78352 Jouy-en-Josas, cedex, France
}

(Reçu le 7 mai 1990; accepté le 30 janvier 1991)

\begin{abstract}
Résumé - Les diarrhées colibacillaires, notamment celles dues à des souches d'E coli entéropathogènes pourvues de l'antigène K88, sont une cause majeure de mortalité chez le porcelet nouveau-né. L'existence d'une résistance génétique, liée à plusieurs marqueurs biochimiques, dont les bases sont brièvement rappelées ici, permet d'envisager de réduire par sélection l'incidence économique de la maladie. Cet article présente les conditions dans lesquelles une telle sélection pourrait s'intégrer dans les schémas courants d'amélioration génétique du porc. La plupart des populations porcines manifestent un polymorphisme pour les gènes de résistance à la colibacillose K88. Dans une population exposée à cette maladie, la mortalité du porcelet dépend de la fréquence $p$ du gène de résistance et de 2 taux de mortalité $m_{1}$ et $m_{2}$, s'appliquant respectivement aux porcelets sensibles issus de mères sensibles, qui bénéficient d'une certaine protection colostrale, et aux porcelets sensibles issus de mères résistantes, qui ne bénéficient pas de cette protection. De ce fait on a généralement $m_{1}<m_{2}$, et on montre que, au sein d'une population, le risque global de mortalité peut, dans certaines conditions, être une fonction croissante de la fréquence du gène de résistance. Dans un système de croisement, le choix d'un génotype approprié pour les verrats utilisés à chaque niveau permet de minimiser la mortalité du porcelet terminal. Ainsi l'utilisation d'un verrat terminal résistant offre l'avantage de la plus faible mortalité dans sa descendance, quelle que soit la fréquence $p$ chez les mères, et cette mortalité diminue à mesure que $p$ augmente. On note, au contraire, que si le verrat terminal est homozygote sensible, la mortalité augmente avec $p$. Dans un système de croisement triple, par exemple, l'utilisation d'un grand-père maternel homozygote sensible devrait alors être recommandée. Il faut aussi prendre en compte les effets de la sélection naturelle, par la mortalité, et de la sélection (artificielle) sur l'âge à $100 \mathrm{~kg}$, qui peuvent, dans certaines situations, défavoriser le gène de résistance. On montre cependant que la fréquence $p=0,5$ est un seuil critique, au-dessus duquel ce risque est totalement écarté. L'existence de 3 variants sérologiques de l'adhésine K88 et de 3 récepteurs intestinaux correspondants apporte une complexité supplémentaire, et peut induire des interactions entre plusieurs locus de résistance. En tout état de cause, un important travail d'identification des génotypes est nécessaire, au moins dans les lignées
\end{abstract}


paternelles. Des recherches restent à poursuivre pour parvenir à un marquage génétique suffisamment étroit des gènes de résistance ou à leur identification biochimique.

résistance génétique aux maladies / porc / K88 / schéma de croisement / sélection naturelle / gène marqueur

Summary - Use of genetic resistance to K88 colibacillosis in pig breeding schemes. Neonatal colibacilloses, and particularly those due to strains of $\mathrm{E}$ coli bearing the K88 antigen, are a major cause of piglet mortality. The existence of a genetic resistance, linked to several biochemical markers, which is briefly presented here, offers the possibility of reducing the economic impact of the disease through selection. This paper discusses the conditions under which such a selection could be implemented within current pig breeding schemes. Most pig populations are polymorphic with regard to the gene(s) for resistance to K88 colibacillosis. The adhesion phenotypes for the 3 possible variants, K88ab, K88ac, and K88ad, are given in table $I$, and their observed frequencies in 5 reports from the literature are given in table II. Within a population exposed to the disease, piglet mortality depends on the frequency $\mathrm{p}$ of the resistance gene and on 2 mortalities $\mathrm{m}_{1}$ and $\mathrm{m}_{2}$, respectively applying to susceptible pigs from susceptible dams, which benefit from colostral protection, and to susceptible pigs from resistant dams, which lack this protection. From table III, which gives the expected mortality for the different combinations of sire and dam genotype, the global risk of mortality may be derived in a population in Hardy-Weinberg equilibrium and expressed as a function of $\mathrm{p}$. As shown in figure 1 , in which mortality is plotted against $\mathrm{p}$ for various values of $\mathrm{m}_{1}$ and $\mathrm{m}_{2}$, the risk of mortality may increase with $\mathrm{p}$, under some conditions. In a crossbreeding scheme, the choice of an appropriate boar genotype at each level allows minimizing mortality among terminal piglets. The use of a resistant terminal sire, for instance, minimizes mortality in the progeny, whichever the frequency $\mathrm{p}$ in the dam line(s), and mortality decreases with increasing $\mathrm{p}$. However, as shown in figure 2, where mortality is plotted against gene frequency in the dam line for each sire genotype, if the terminal sire is homozygous susceptible, mortality in the progeny increases with p. In a 3-way cross, the use of a homozygous susceptible maternal grand-sire should then be recommended, as shown in table $I V$, which gives the expected mortality for each combination of sire and maternal grand-sire genotype. In addition, natural selection, through piglet mortality, and (artificial) selection for age at $100 \mathrm{~kg}$ are also to be considered, as they may, in some situations, act against the resistance gene. However, it can be shown that the gene frequency value $\mathrm{p}=0.5$ is a critical threshold, above which such a risk is totally avoided. This is shown graphically for natural selection in figure 3, which gives the gene frequency change due to mortality as a function of the gene frequency in the population. The existence of 3 serological variants of the K88 adhesin and of 3 corresponding intestinal receptors introduces additional complexities and it may induce interactions between several receptor loci. In any case, a large amount of work for identifying genotypes is necessary, at least in sire lines. Investigations should be pursued in order to reach a sufficintly precise marking of the resistance genes or to identify them biochemically.

genetic resistance to disease / pig / K88 / crossbreeding scheme / natural selection / marker gene

\section{INTRODUCTION}

Les diarrhées colibacillaires dues à des souches entéropathogènes de Escherichia coli, et plus particulièrement celles pourvues de l'antigène $\mathrm{K} 88$, sont une cause majeure de mortalité chez le porcelet nouveau-né. Des souches porteuses d'autres antigènes 
(987P, K99 et F41 notamment) sont également responsables, mais les enquêtes réalisées en élevage montrent que les colibacilles porteurs de K88 prédominent généralement dans la flore des porcelets diarrhéiques, et que les souches à K88 peuvent même représenter dans certains cas plus de la moitié des souches isolées (voir la revue bibliographique récente de Edfors-Lilja, 1991). Cette maladie exerce par ailleurs des effets défavorables sur la croissance des porcelets qui survivent aux épisodes de diarrhée. Waldmann (1990) observe par exemple une réduction de poids de $30 \%$ à $10 \mathrm{j}$ chez ces derniers par rapport à des porcelets sains depuis la naissance, dans le même troupeau expérimental. L'existence d'une résistance génétique à cette maladie, qui était soupçonnée depuis longtemps (voir les références anciennes données par Rapacz et Hasler-Rapacz, 1986), a été démontrée par Sellwood et al (1975). On peut donc envisager, à côté d'une prophylaxie médicale, l'utilisation de moyens génétiques pour réduire l'incidence économique de la maladie, comme cela a été discuté par Sellwood (1979, 1980) et Ollivier et al (1984). L'objet de cet article est de présenter les possibilités d'utilisation de cette résistance génétique dans le cadre des schémas d'amélioration génétique couramment appliqués chez le porc.

\section{BASE GÉNÉTIQUE DE LA RÉSISTANCE À LA COLIBACILLOSE K88}

La colonisation de l'intestin, nécessaire pour que la maladie apparaisse, dépend d'un antigène spécifique qui permet l'adhésion des bactéries à la paroi intestinale. L'attachement des souches de $E$ coli K88 aux cellules épithéliales de l'intestin grêle implique des récepteurs localisés sur la membrane des bordures en brosse. L'attachement peut être mis en évidence soit directement in vivo (Sellwood et al, 1975; Snodgrass et al, 1981), soit indirectement in vivo par des numérations bactériennes du contenu et des parois de l'intestin après inoculation de porcelets axéniques (Chappuis et al, 1984).

Si la nature biochimique exacte des récepteurs n'est pas pour le moment élucidée, il est établi qu'ils sont transmis comme des facteurs mendéliens simples, le caractère non adhésif étant récessif (gène $s$ ) par rapport au caractère adhésif (gène $S$ ). Les 2 phénotypes, attachant (A) et non attachant (NA), correspondent donc à 3 génotypes, SS, Ss et ss (Gibbons et al, 1977). Si on considère les 3 récepteurs correspondant aux 3 variants connus de K88 (K88ab, K88ac et K88ad), le nombre des phénotypes possibles est théoriquement de 8 . En fait, 6 phénotypes seulement ont jusqu'à présent été dénombrés, comme indiqué au tableau I.

Plusieurs hypothèses ont été avancées pour expliquer la base génétique de l'attachement des différents variants (Rapacz et Hasler-Rapacz, 1986; Bijlsma et Bouw, 1987; Duval-Iflah et al, 1987b). Des résultats récents confirment l'existence de 2 locus distincts contrôlant les récepteurs K88ab et K88ac. Ces 2 locus sont étroitement liés entre eux et ils sont liés par ailleurs au locus Tf de la transferrine. L'ordre le plus probable des 3 locus est Tf-K88ab-K88ac et les distances ont été estimées à 10,9 et 3,6 centimorgans respectivement entre Tf et K88ab et entre K88ab et K88ac (Bonneau et al, 1990). Ces 3 locus appartiennent en fait à un groupe de liaison qui comprend maintenant 6 locus au total. Il faut en effet y inclure également un facteur de létalité précoce dont la liaison avec Tf a été démontrée par Imlah (1970), le locus de la céruloplasmine que Juneja et al (1989) situent à moins de 
Tableau I. Classification des phénotypes d'adhésion selon les différents variants de K88. + : adhésion; - : non-adhésion ; 1 : classification selon Bijlsma et al (1982); 2 : classification selon Rapacz et Hasler-Rapacz (1986); 3 : classification selon Duval-Iflah et al (1987a) : sont seulement considérés les 2 variants K88ab et K88ac.

\begin{tabular}{cccccc}
\hline \multicolumn{3}{c}{ Test d'adhésion } & \multicolumn{3}{c}{ Phénotypes } \\
K88ab & K88ac & K88ad & 1 & 2 & 3 \\
\hline+ & + & + & A & IV & \\
+ & + & - & B & III & ++ \\
+ & - & + & C & & +- \\
- & - & + & D & II & \\
- & - & - & E & I & \\
- & + & - & - & IIc & -+ \\
\hline
\end{tabular}

8 centimorgans de $\mathrm{Tf}$, et le groupe sanguin $\mathrm{L}$ dont le taux de recombinaison avec les locus contrôlant les récepteurs K88 a été récemment évalué à 22,7 centimorgans par Vögeli et al (1990). L'hypothèse d'un locus distinct, lié aux précédents, contrôlant le récepteur K88ad est d'autre part avancée par Rapacz et Hasler-Rapacz (1986) et par Bijlsma et Bouw (1987). Mais le déterminisme du caractère K88ad est rendu difficile à élucider par suite d'une affinité variable, et souvent réduite, entre cet antigène et son récepteur. Un effet de l'âge sur cette affinité a par exemple été récemment suggéré par $\mathrm{Hu}$ et al (1990).

La plupart des populations porcines manifestent un polymorphisme génétique, avec des fréquences d'individus résistants variables selon les troupeaux (Walters et Sellwood, 1982) et selon les races (voir la revue de Edfors-Lilja, 1991). Il faut noter en particulier que certaines races chinoises pourraient être homozygotes résistantes (ss). Dans la race Meishan par exemple, aucun individu sensible (pour K88ab et K88ac) n'a jusqu'à présent été trouvé dans l'échantillon de cette race importé en France, que ce soit par le test in vivo ou le test in vitro (Duval-Iflah et al, 1990). Cette résistance a par ailleurs été confirmée après inoculation de porcelets par voie orale en élevage holoxénique (Duchet-Suchaux et Bertin, 1987). Il semble en outre que les facteurs d'attachement pour les 2 antigènes K88ab et K88ac s'associent de manière préférentielle, les phénotypes $(++)$ et $(--)$ étant prédominants par rapport aux $(+-)$ et $(-+)$, comme indiqué dans le tableau II.

\section{RELATION ENTRE LA MORTALITÉ GLOBALE ET LA FRÉQUENCE DU GÉNE DE RÉSISTANCE DANS UNE POPULATION EN ÉQUILIBRE DE HARDY-WEINBERG EXPOSÉE À UN TYPE DE COLIBACILLOSE K88}

Considérons le cas d'une population exposée à une colibacillose due à une souche bactérienne pourvue d'un antigène donné, par exemple K88ab. Le risque de mortalité du porcelet dépend à la fois de son génotype propre et du génotype de sa 
Tableau II. Distribution des phénotypes d'adhésion pour les variants K88ab et K88ac. 1 Bijlsma et al (1982) : prélèvements en abattoir sur animaux d'origine inconnue; 2 Rapacz et Hasler-Rapacz (1986) : animaux purs ou croisés représentant 7 races américaines; 3 Bijlsma et Bouw (1987) : croisements entre Landrace et Large White; 4 Gautschi et Schwörer (1989) : animaux de race Large White, Landrace suisse et Hampshire, appartenant à des élevages de sélection suisses; $5 \mathrm{Hu}$ et al (1990) : 268 porcs d'origine non précisée, répartis en $n_{1}(++)$ et $n_{2}(--)$ avec des valeurs de $n_{1}$ et $n_{2}$ non données.

\begin{tabular}{|c|c|c|c|c|c|c|}
\hline \multicolumn{2}{|c|}{ Test d'adhésion } & \multicolumn{5}{|c|}{ Effectifs observés } \\
\hline$K 88 a b$ & $K 88 a c$ & 1 & 2 & 3 & 4 & 5 \\
\hline+ & + & 31 & 172 & 10 & 212 & $n_{1}$ \\
\hline+ & - & 10 & 0 & 1 & 0 & 0 \\
\hline- & - & 24 & 171 & 9 & 253 & $n_{2}$ \\
\hline- & + & 0 & 2 & 0 & 0 & 0 \\
\hline
\end{tabular}

mère pour le gène de résistance correspondant. Gibbons et al (1977) et Sellwood (1982) ont en effet montré que le colostrum des truies de phénotype attachant (SS ou Ss) confère une protection du porcelet plus efficace que celui des mères de phénotype résistant (ss). La mortalité dépend alors de la fréquence $p$ du gène de résistance dans la population et des taux de mortalité $m_{1}$ et $m_{2}$ s'appliquant aux porcelets sensibles issus respectivement de mères sensibles (individus protégés par le colostrum) et de mères résistantes (individus non protégés par le colostrum). Les mortalités moyennes correspondant aux 9 types de portées qui peuvent se présenter sont données en fonction des valeurs de $m_{1}$ et $m_{2}$ dans le tableau III avec les moyennes marginales correspondant aux fréquences génotypiques de la loi de Hardy-Weinberg. On en déduit une expression de la mortalité moyenne $(M)$ d'une population en fonction de $p(q=1-p), m_{1}$ et $m_{2}$ :

$$
M=q\left[p^{2}\left(m_{2}-m_{1}\right)+p m_{1}+m_{1}\right]
$$

Tableau III. Taux de mortalité par colibacillose des porcelets en fonction des génotypes du père et de la mère dans une population en équilibre. $p:$ fréquence du gène de résistance; $m_{1}$ : taux de mortalité des porcelets sensibles protégés par le colostrum maternel; $m_{2}$ : taux de mortalité des porcelets sensibles non protégés par le colostrum maternel.

\begin{tabular}{lccccc}
\hline $\begin{array}{l}\text { Génotype } \\
\text { de la }\end{array}$ & & \multicolumn{3}{c}{ Génotype du père } & $\begin{array}{c}\text { Moyennes } \\
\text { màre }\end{array}$ \\
\cline { 3 - 5 } & Fréquence & $s s$ & $s S$ & $S S$ & \\
& génotypique & $p^{2}$ & $2 p q$ & $q^{2}$ & \\
\hline $\mathrm{ss}$ & $p^{2}$ & 0 & $0,5 m_{2}$ & $m_{2}$ & $m_{2} q$ \\
$\mathrm{sS}$ & $2 p q$ & $0,5 m_{1}$ & $0,75 m_{1}$ & $m_{1}$ & $0,5 m_{1}(1+q)$ \\
$\mathrm{SS}$ & $q^{2}$ & $m_{1}$ & $m_{1}$ & $m_{1}$ & $m_{1}$ \\
Moyennes & marginales & $m_{1}(1-p)$ & $0,5 p^{2}\left(m_{2}-m_{1}\right)$ & $p^{2}\left(m_{2}-m_{1}\right)+m_{1}$ & Équation (1) \\
& & & $-0,5 m_{1} p+m_{1}$ & & \\
\hline
\end{tabular}


La figure 1 montre l'évolution de $M$ pour diverses valeurs de $m_{1}$ et $m_{2}$, sachant qu'on a, par hypothèse, $m_{2}>m_{1}$. On voit que plus l'écart entre $m_{1}$ et $m_{2}$ se réduit, plus on tend vers une relation «normale» entre $M$ et $p$, c'est-à-dire une diminution de la mortalité quand la fréquence du gène de résistance augmente. Dans le cas extrême où $m_{1}=0$ (mortalité nulle des individus sensibles issus des mères sensibles) et $m_{2}=1$ (mortalité totale des individus sensibles issus des mères résistantes) on obtient $M=p^{2} q$ et on constate, comme la figure 1 le montre, que jusqu'à une fréquence $p=2 / 3$, le risque de mortalité augmente avec la fréquence du gène de résistance dans la ponulation.

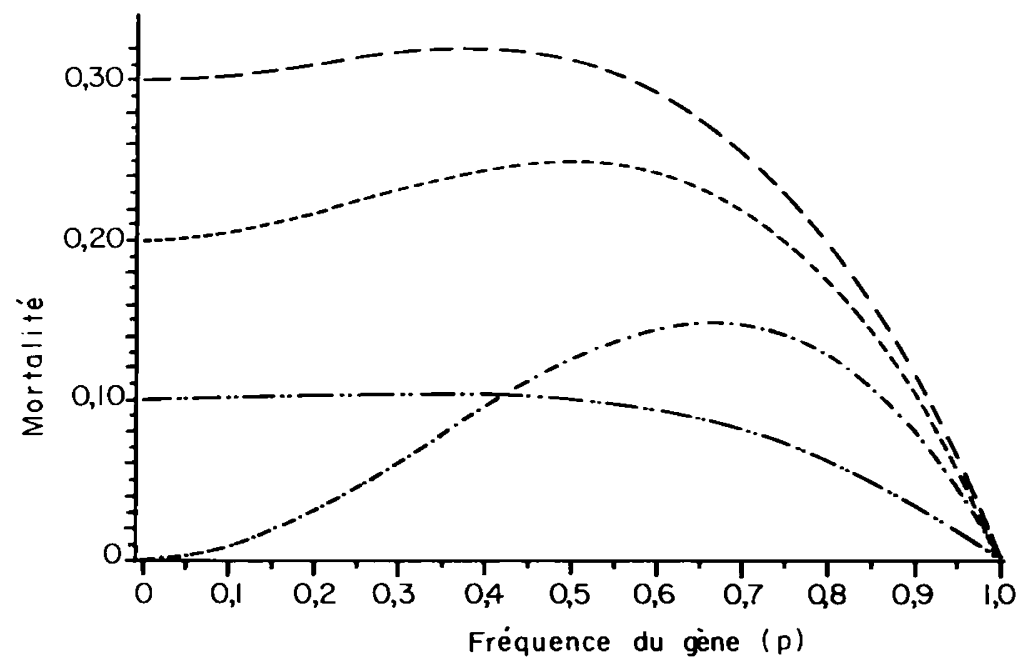

Fig 1. Mortalité moyenne due à la colibacillose K88 en fonction de la fréquence du gène de résistance $(p)$ dans une population en équilibre. $-\cdots \cdot-\cdot-\cdot m_{1}=0 ; m_{2}=1$; $-\ldots-m_{1}=0,2 ; m_{2}=1 ;-\ldots-\ldots m_{1}=0,3 ; m_{2}=1 ;-\cdots-\cdots-\cdots m_{1}=$ 0,$1 ; m_{2}=0,3 ; m_{1}$ et $m_{2}$ définis au tableau III.

\section{ÉVOLUTION DE LA MORTALITÉ MOYENNE DANS DIFFÉRENTS SYSTĖMES DE CROISEMENT}

\section{Croisement simple}

Dans ce type de croisement on suppose que, pour le locus de résistance, la lignée maternelle est en équilibre de Hardy-Weinberg et que les mâles utilisés ont un génotype défini, soit SS, Ss ou ss. On peut donc utiliser les moyennes marginales du tableau III pour représenter l'évolution de la mortalité moyenne des produits de croisement en fonction de la fréquence $p$ dans la lignée maternelle (fig 2). Cette évolution montre que l'utilisation d'un verrat résistant (ss) est à recommander, puisqu'il donne la plus faible mortalité en croisement quel que soit le génotype 
de la lignée maternelle, comme le remarquait déjà Sellwood (1979). On voit aussi que, si un verrat ss est utilisé, la mortalité diminue quand la fréquence $p$ du gène de résistance dans la lignée maternelle augmente, alors que si le verrat est SS la mortalité augmente avec $p$.

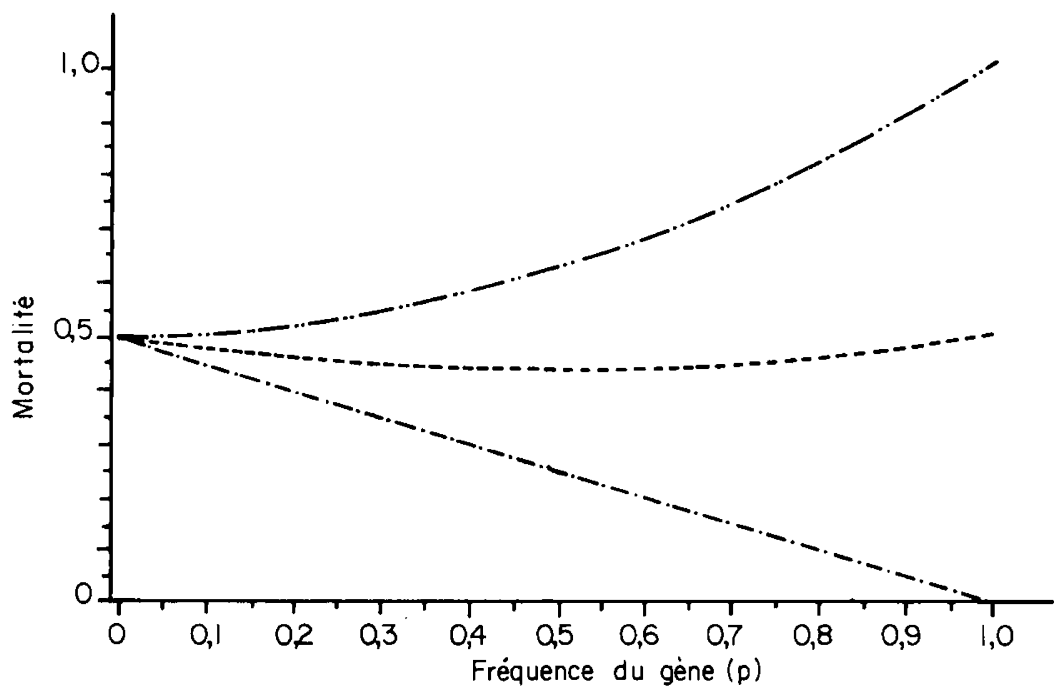

Fig 2. Mortalité moyenne des descendants en fonction du génotype du père et de la fréquence du gène de résistance $(p)$ des mères dans un croisement simple $\left(m_{1}=0,5\right.$; $\left.m_{2}=1\right) ; m_{1}$ et $m_{2}$ définis au tableau III. - . - - - - Père ss; - - - Père Ss; $-\cdots-\cdots$ Père SS.

\section{Croisement triple}

La mortalité du produit terminal va maintenant dépendre des génotypes des 3 lignées utilisées : la lignée grand-maternelle (GMM), la lignée grand-paternelle (GPM) et la lignée paternelle (P). On suppose que la lignée GMM est en équilibre de Hardy-Weinberg pour le gène de résistance, et que les mâles GPM et $P$ utilisés ont un génotype défini, soit $\mathrm{SS}$, Ss ou ss. Il y a donc 9 cas à envisager selon les génotypes GPM et P. Le tableau IV donne la mortalité moyenne des porcelets pour chaque combinaison. On voit que la mortalité évolue toujours linéairement avec la fréquence du gène de résistance dans la lignée GMM. Pour chaque génotype GPM le meilleur verrat terminal est ss, comme dans le cas du croisement simple. Mais si le verrat terminal est homozygote sensible (SS), la mortalité peut être très élevée avec un GPM résistant (ss) et le meilleur GPM à utiliser est alors l'homozygote sensible (SS). 
Tableau IV. Mortalité moyenne des porcelets en fonction des génotypes du père $(P)$ et du grand-père maternel $(\mathrm{GPM})$ dans un système de croisement triple : $\mathrm{P} \times(\mathrm{GPM} \times \mathrm{GMM})$; $p:$ fréquence du gène de résistance dans la lignée grand-maternelle GMM; $m_{1}$ et $m_{2}$ définis au tableau III.

\begin{tabular}{|c|c|c|c|}
\hline \multirow{2}{*}{$\begin{array}{l}\text { Génotype du } \\
\text { grand-père } \\
\text { maternel } \\
\text { (GPM) }\end{array}$} & \multicolumn{3}{|c|}{ Génotype du père $(P)$} \\
\hline & ss & $s S$ & $S S$ \\
\hline $\begin{array}{l}\text { ss } \\
\text { sS } \\
\text { SS }\end{array}$ & $\begin{array}{l}0,5 m_{1}(1-p) \\
0,5 m_{1}(1,5-p) \\
m_{1}(1-0,5 p)\end{array}$ & $\begin{array}{l}0,75 m_{1}+0,5 p\left(m_{2}-1,5 m_{1}\right) \\
0,875 m_{1}+0,25 p\left(m_{2}-2 m_{1}\right) \\
m_{1}(1-0,25 p)\end{array}$ & $\begin{array}{l}m_{1}+p\left(m_{2}-m_{1}\right) \\
m_{1}+0,5 p\left(m_{2}-m_{1}\right) \\
m_{1}\end{array}$ \\
\hline
\end{tabular}

\section{Autres systèmes de croisement}

Les considérations précédentes peuvent s'appliquer à tout système de croisement. On voit que le caractère récessif de la résistance rend son utilisation relativement complexe. Si le verrat terminal est croisé, par exemple, les 2 races ou lignées servant à le produire devraient être de génotype ss. À défaut, une sélection du verrat terminal sur la base d'un test d'attachement est souhaitable. Il y a alors avantage à maintenir dans ces lignées la fréquence du gène de résistance à un niveau aussi élevé que possible. Mais toute sélection en faveur de ce gène doit prendre en compte l'ensemble des autres facteurs susceptibles de faire évoluer sa fréquence au sein des lignées utilisées. Ce point va être abordé dans ce qui suit.

\section{EFFETS DE LA SÉLECTION DANS UNE POPULATION FERMÉE}

\section{Sélection naturelle}

Gibbons et al (1977) ont montré que dans les conditions d'élevage exposant les animaux à la maladie, les relations décrites plus haut entre la mortalité et le génotype, tant individuel que maternel, induisent un système génétique à coefficients de sélection variables qui aboutit à un équilibre instable. En adaptant les formules de Gibbons et al (1977) en fonction des mortalités $m_{1}$ et $m_{2}$ précédemment définies, les fréquences et les mortalités des différents génotypes, dans les conditions de l'équilibre de Hardy-Weinberg, sont représentées au tableau V.

Tableau V. Fréquence et mortalité des différents génotypes dans une population en équilibre.

\begin{tabular}{lcc}
\hline Génotype & Fréquence & Mortalité \\
\hline$s s$ & $p^{2}$ & 0 \\
$s S$ issus de mères ss & $p^{2}(1-p)$ & $m_{2}$ \\
$s S$ autres & $\left(2 p-p^{2}\right)(1-p)$ & $m_{1}$ \\
$S S$ & $(1-p)^{2}$ & $m_{1}$ \\
\hline
\end{tabular}


On voit que la mortalité moyenne des hétérozygotes est égale à $m_{1}+p\left(m_{2}-\right.$ $\left.m_{1}\right) / 2$. Comme $m_{2}>m_{1}$, les hétérozygotes sont en moyenne soumis à une mortalité supérieure à celle des homozygotes. Avec des valeurs sélectives égales à $1-m_{1}$ et $1-m_{2}$, on montre que le système conduit à un changement de fréquence génique $\Delta p$ par génération tel que :

$$
\Delta p=p^{2} q\left[2 p\left(m_{2}-m_{1}\right)+3 m_{1}-m_{2}\right] / 2\left\{1-m_{2} p^{2} q-m_{1}\left[\left(2 p-p^{2}\right) q+q^{2}\right]\right\}
$$

Il existe donc une fréquence d'équilibre $p_{0}$ (différente de 0 et 1 ), pour laquelle $\Delta p=0$, soit :

$$
p_{0}=0,5-m_{1} /\left(m_{2}-m_{1}\right)
$$

La figure 3 montre l'évolution de $\Delta p$ pour différentes valeurs de $m_{1}$ et $m_{2}$. Comme le montre l'expression (3), la fréquence d'équilibre maximale est 0,5 et cette fréquence diminue au fur et à mesure que le rapport $m_{2} / m_{1}$ diminue. Il s'agit d'un équilibre instable et le risque existe donc que le gène de résistance soit éliminé naturellement, lorsque le rapport $m_{2} / m_{1}$ qui prévaut dans la population conduit à des valeurs négatives de $\Delta p$. Ce risque est toutefois exclu si $p_{0}>0,5$, quelles que soient les valeurs de $m_{1}$ et $m_{2}$. Par ailleurs, la possibilité d'un équilibre instable suppose aussi que $p_{0}>0$, ce qui correspond à $m_{2} / m_{1}>3$.

On peut noter par ailleurs que le mécanisme sélectif qui vient d'être décrit, qui implique un désavantage de l'hétérozygote, est similaire à celui du système Rhésus $D / d$ chez l'homme. Dans le cas particulier où $m_{1}=0$, l'équation (2) est en effet identique à celle donnée par Cavallis-Sforza et Bodmer (1971, p 199) pour la fréquence du gène $d$.

\section{Sélection artificielle}

L'âge à $100 \mathrm{~kg}$ est un critère de sélection couramment appliqué dans le choix des reproducteurs porcins. Dans la mesure où ce critère est influencé par les diarrhées néo-natales (voir, par exemple, Hoy et Hörügel, 1985, qui attribuent à ces diarrhées un effet défavorable de plus de $3 \%$ ), la fréquence du gène de résistance se trouve influencée par ce type de sélection (artificielle). En supposant que l'âge à $100 \mathrm{~kg}$ est accru de $a_{1} \mathrm{j}$ chez les individus sensibles protégés par le colostrum maternel et de $a_{2} \mathrm{j}$ chez les individus sensibles non protégés par le colostrum maternel, les valeurs génotypiques pour l'âge à $100 \mathrm{~kg}$ (en écart à celui des individus résistants) s'expriment en fonction de $a_{1}$ et $a_{2}$ comme les valeurs de mortalité indiquées au tableau $\mathrm{V}$ en fonction de $m_{1}$ et $m_{2}$. En particulier, la valeur génotypique moyenne des hétérozygotes est égale à $a_{1}+p\left(a_{2}-a_{1}\right) / 2$, et, comm $a_{2}>a_{1}$, les hétérozygotes ont toujours une valeur supérieure à celle des homozygotes. Par ailleurs, l'effet moyen de la substitution de $\mathrm{s}$ à $\mathrm{S}$ s'écrit :

$$
-p\left(a_{2}-a_{1}\right)\left[p-0,5+a_{1} /\left(a_{2}-a_{1}\right)\right]
$$

On voit que cet effet est négatif, ce qui veut dire que la sélection en faveur du gène de résistance s tend à diminuer l'âge à $100 \mathrm{~kg}$, à condition que $p>0,5-a_{1} /\left(a_{2}-a_{1}\right)$. Réciproquement, si cette condition est remplie, la sélection pour diminuer l'âge à $100 \mathrm{~kg}$ aura pour effet d'augmenter la fréquence du gène de résistance. Mais, si cette condition n'est pas remplie, une diminution de la fréquence du gène est à prévoir. On voit que ce risque est cependant exclu si $p<0,5$, quelles que soient les valeurs de $a_{1}$ et de $a_{2}$. 


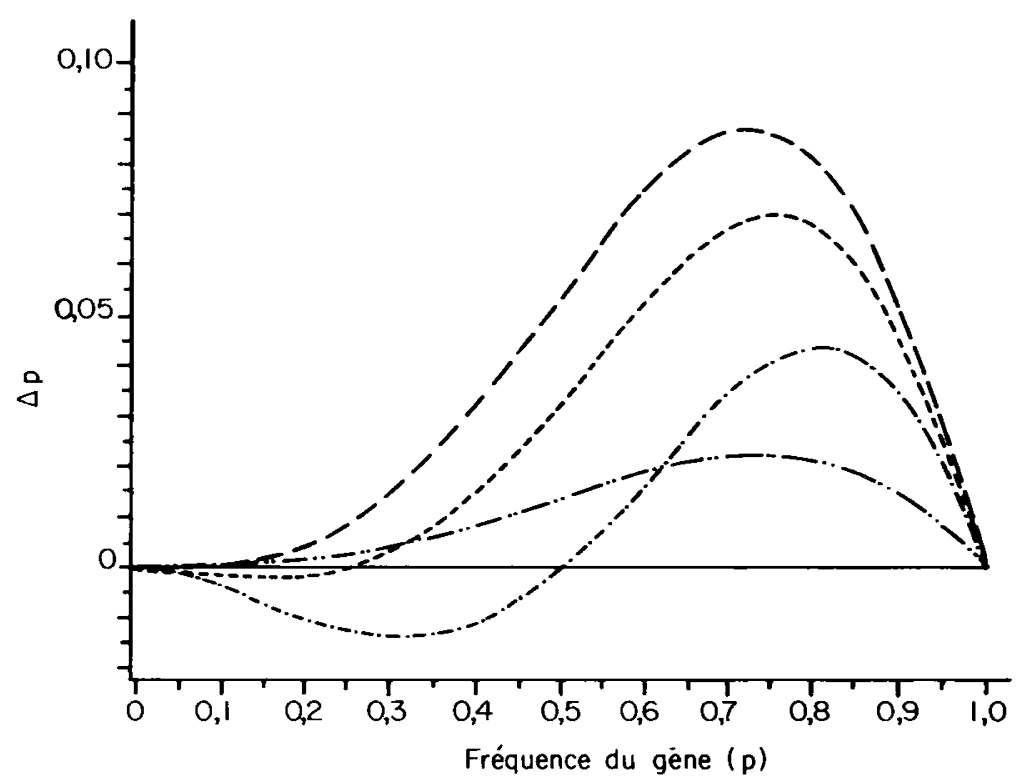

Fig 3. Changement par génération $(\Delta p)$ de la fréquence du gène de résistance en fonction de sa fréquence $(p)$ dans la population. $-\cdot \cdots \cdot \cdot-\cdot m_{1}=0 ; m_{2}=1 ;-\cdots-\cdots \cdots m_{1}=$ 0,$1 ; m_{2}=0,3 ; m_{1}$ et $m_{2}$ définis au tableau III; $---m_{1}=0,2 ; m_{2}=1$; $---m_{1}=0,3 ; m_{2}=1$.

\section{DISCUSSION ET CONCLUSIONS}

Une sélection pour la résistance aux colibacilloses K88 est réalisable et, depuis déjà quelques années, elle est effectivement mise en œuvre dans la pratique (voir Sellwood, 1980). Comme nous l'avons vu ci-dessus, cette sélection doit être adaptée au système de croisement utilisé et, dans les schémas les plus complexes, un important travail d'identification des génotypes résistants est nécessaire, au moins dans les lignées paternelles.

Il faut aussi tenir compte des forces de sélection, naturelle ou indirecte, qui peuvent s'opposer à la sélection directe des gènes de résistance. Comme indiqué ci-dessus, on note que la fréquence génique de 0,5 pour le gène de résistance est une valeur critique, au-dessous de laquelle la disparition du gène est un risque à envisager, sous l'effet direct de la sélection naturelle par la mortalité ou sous l'effet indirect de la sélection pour la vitesse de croissance. Le risque existe aussi que des effets de dérive génétique entrainent dans un élevage une baisse fortuite de la fréquence des gènes de résistance. Il faut remarquer cependant que ce risque d'élimination des gènes favorables suppose que la population ait été exposée à la maladie sur une période suffisamment longue pour que les truies aient acquis une certaine immunité induisant 2 taux de mortalité $m_{1}$ et $m_{2}$ assez différents. 
Il faut souligner que notre étude concerne un locus de résistance considéré isolément, avec un mécanisme sélectif indépendant du génotype à d'autres locus. Mais l'existence des 3 variants sérologiques de l'adhésine K88 introduit une complexité supplémentaire, d'autant plus que la base génétique sous-jacente reste encore incomplètement élucidée. Si on se limite aux 2 variants K88ab et K88ac, les résultats du tableau II, bien que basés sur des effectifs limités et un matériel génétiquement très hétérogène, indiquent une tendance à des associations préférentielles entre les phénotypes à ces 2 locus. Cela suggère l'existence d'un mécanisme sélectif s'exerçant à l'encontre des individus résistants à l'un seulement des 2 variants. Ce mécanisme pourrait s'expliquer à la lumière des résultats de Bijlsma et al (1987), qui montrent qu'une immunité colostrale à l'égard des 2 variants K88ab et K88ac se manifeste chez des mères ne possédant que l'un des récepteurs. Pour qu'un porcelet sensible ne bénéficie d'aucune protection colostrale, il est donc nécessaire que sa mère soit double homozygote résistante. Il s'établit alors un système de sélection à 2 locus épistatiques et, dans le cas particulier d'une population en équilibre, on peut montrer que la proportion des mères doublement résistantes chez les porcelets hétérozygotes sensibles pour l'un des variants et résistants pour l'autre est le double de celle que l'on trouve chez les porcelets doubles hétérozygotes. Cela pourrait expliquer le désavantage sélectif des premiers, de phénotype $(+-)$ ou $(-+)$, par rapport aux seconds, de phénotype $(++)$. L'évolution d'un tel système est cependant difficile à prévoir, étant donné que les coefficients de sélection aux 2 locus dépendent des fréquences géniques et de l'association gamétique qui peut s'instaurer entre eux, et aussi de la fréquence relative et de la virulence des 2 souches bactériennes impliquées.

Sur un plan plus général, l'intérêt d'une sélection pour cette résistance dépend d'abord de la fréquence et de la stabilité de l'agent pathogène. Comme nous l'avons indiqué dans l'introduction, les souches bactériennes porteuses des antigènes K88 sont largement répandues. Elles ont également été identifiées depuis longtemps dans plusieurs pays et rien n'indique pour le moment qu'elles aient évolué vers des types nouveaux. Une sélection pour cette résistance est donc économiquement justifiée dans une perspective de réduction de la mortalité néo-natale. Il reste par ailleurs à s'assurer que les gènes ainsi sélectionnés n'ont pas d'effets négatifs sur les caractères de production. Des résultats concordants quant à l'effet néfaste des récepteurs K88 sur la croissance du porcelet avant le sevrage ont été rapportés par Edfors-Lilja et al (1986) et par Gautschi et Schwörer (1989). Ces résultats pourraient être la conséquence de diarrhées plus fréquentes chez les porcelets porteurs des récepteurs. Quant aux performances d'engraissement et de carcasse, à côté des résultats de Edfors-Lilja et al (1986) qui indiquent une croissance musculaire plus rapide des porcelets sensibles, il faut mentionner ceux de Walters et Sellwood (1987) et de Gautschi et Schwörer (1989) qui ne confirment pas les précédents. Les comparaisons effectuées jusqu'à présent sont encore peu nombreuses et elles méritent d'être poursuivies pour mieux établir les effets de ces gènes.

Sur le plan pratique, la possibilité de détecter facilement les individus résistants est une condition essentielle pour le succès de ce type de sélection. Une étape décisive, de ce point de vue, fut la mise au point de la technique in vitro et son application à des fragments d'intestin prélevés par biopsie (Sellwood et al, 1975; Snodgrass et al, 1981). Mais ces techniques restent encore relativement lourdes à 
mettre en œuvre. L'utilisation de gènes marqueurs, faciles à identifier, est une voie à explorer et les liaisons récemment trouvées entre les locus des récepteurs K88 et plusieurs marqueurs biochimiques ouvrent des perspectives intéressantes. Il reste cependant à démontrer l'existence d'associations gamétiques entre ces locus et, de ce point de vue, les distances estimées, de l'ordre de $10 \%$, ne sont pas a priori favorables à l'établissement d'associations préférentielles. Les recherches doivent être poursuivies en vue de réaliser un marquage plus étroit des gènes concernés et de parvenir éventuellement à leur identification biochimique.

\section{REMERCIEMENTS}

Les auteurs remercient $\mathrm{S}$ Nugier et $\mathrm{H}$ Jouet pour leur aide dans la réalisation de ce travail.

\section{RÉFÉRENCES}

Bijlsma IGW, De Nijs A, Van Der Meer C, Frik JF (1982) Different pig phenotypes affect adherence of Escherichia coli to jejunal brush borders by K88ab, K88ac, or K88ad antigen. Infect Immun 37, 891-894

Bijlsma IGW, Bouw J (1987) Inheritance of K88-mediated adhesion of Escherichia coli to jejunal brush borders in pigs : a genetic analysis. Vet Res Commun 11, 509-518

Bijlsma IGW, Van Houten M, Frik JF, Ruitenberg EG (1987) K88 variants K88ab, $\mathrm{K} 88 \mathrm{ac}$ and $\mathrm{K} 88 \mathrm{ad}$ in oral vaccination of different porcine adhesive phenotypes. Immunological aspects. Vet Immunol Immunopathol 16, 235-250

Bonneau M, Duval-Iflah Y, Guérin G, Ollivier L, Renard C, Renjifo X (1990) Aspects génétiques et microbiologiques de la colibacillose K88 chez le porc. Ann Rech Vét 21, 302-303 (résumé)

Cavalli-Sforza LL, Bodmer WF (1971) The Genetics of Human Populations. Freeman, San Francisco, CA

Chappuis JP, Duval-Iflah Y, Ollivier L, Legault C (1984) Escherichia coli K88 adhesion : a comparison of Chinese and Large White piglets. Génét Sél Evol 16, 385-390

Duchet-Suchaux M, Bertin A (1987) Comparaison de la sensibilité de porcelets Large White et Meishan à différentes souches de colibacilles entérotoxinogènes. Ann Rech Vét 18, 334-335

Duval-Iflah Y, Guérin G, Renard C, Ollivier L (1987a) K88 colibacillosis in pig : preliminary genetic results. $38^{e}$ Réunion Annuelle, Féd Eur Zootech, Lisbonne, Portugal, 28 sept-1 $1^{\text {er }}$ oct $1987,6 \mathrm{p}$

Duval-Iflah Y, Ollivier L, Guérin G, Bonneau M, Caritez JC, Renard C (1987b) La colibacillose néo-natale K88 du porc. Résultats génétiques préliminaires. Ann Rech Vét 18, 333-334

Duval-Iflah Y, Ouriet MF, Guillaume P, Caritez JC, Ollivier L (1990) Étude de l'attachement in vivo et in vitro de Escherichia coli K88 chez des porcelets chinois : conséquences sur la colibacillose en conditions gnotoxéniques. In : Symposium sur le Porc Chinois, (Molénat M, Legault C, eds), INRA, Paris, 181 (résumé) 
Edfors-Lilja J (1990) Escherichia coli resistance in pigs. In : Breeding for Disease Resistance in Farm Animals. (Owen JB, Axford RFE, eds), CAB International Wallingford, 424-435

Edfors-Lilja J, Petersson H, Gahne B (1986) Performance of pigs with and without the intestinal receptor for Escherichia coli K88. Anim Prod 42, 381-387

Gautschi C, Schwörer D (1989) An analysis of the intestinal receptor for $E$ coli K88 in different Swiss pig breeds. Anim Genet 20 suppl 1, 38-39

Gibbons RA, Sellwood R, Burrows M, Hunter PA (1977) Inheritance of resistance to neonatal Ecoli diarrhoea in the pig : examination of the genetic system. Theor Appl Genet 51, 65-70

Hoy S, Hörügel K (1965) Zu den Auswirkungen des Saugferkel-Frühdurchfalles auf die Körpermasseentwicklung bis zur Schlachtung. Mh Vet-Med 40, 86-89

Hu ZL, Hasler-Rapacz J, Huang SC, Rapacz J (1990) Studies on inheritance and variations in expression of swine small intestinal receptors for enteropathogenic Escherichia coli K88ad antigen. XXII Int Conf Anim Genet, 25-31 août 1990, East Lansing, Michigan, résumé 2.3 .4

Imlah P (1970) Evidence for the Tf locus being associated with an early lethal factor in a strain of pigs. Anim Blood Groups Biochem Genet 1, 5-13

Juneja RK, Kuryl J, Gahne B, Zurkowski M (1989) Linkage between the loci for transferrin and ceruloplasmin in pigs. Anim Genet 20, 307-311

Ollivier L, Giradeau JP, Duval Y, Chappuis JP (1984) Perspectives de sélection de porcelets résistants à la colibacillose néonatale K88. In : Physiologie et pathologie périnatales chez les animaux de ferme (Jarrige R, ed) INRA, Paris, 465-474

Rapacz J, Hasler-Rapacz J (1986) Polymorphism and inheritance of swine small intestinal receptors mediating adhesion of three serological variants of Escherichia coli-producing K88 pilus antigen. Anim Genet 17, 305-321

Sellwood R (1979) Escherichia coli diarrhoea in pigs with or without the K88 receptor. Vet Rec 105, 228-230

Sellwood R (1980) Breeding for E coli resistance. Pig Farming 28, 72

Sellwood R (1982) Escherichia coli-associated porcine neonatal diarrhoea : antibacterial activities of colostrum from genetically susceptible and resistant sows. Infect Immun 35, 396-401

Sellwood R, Gibbons RA, Jones GW, Rutter JM (1975) Adhesion of enteropathogenic Escherichia coli to pig intestinal brush borders : the existence of two pig phenotypes. J Med Microbiol 8, 405-411

Snodgrass DR, Chandler DA, Makin TJ (1981) Inheritance of Escherichia coli K88 adhesion in pigs : identification of nonadhesive phenotypes in a commercial herd. Vet Rec 109, 461-463

Vögeli P, Kuhn B, Obrist R, Stranzinger G (1990) Genetic linkage for some blood groups, red cell enzymes and serum proteins, and intestinal receptors mediating adhesion of Escherichia coli-producing K88 pilus antigen. XXII Int Conf on Anim Genet, 25-31 août 1990, East Lansing, Michigan, résumé 6.1.6

Waldmann KH (1990) Influence of colibacillosis on fluid, electrolyte and energy balance in newborn pigs. Pig News Inf 11, 337-340 
Waltcrs JR, Sellwood R (1982) Aspect of genetic resistance to K88 E coli in pigs. In : 2nd World Congress on Genetics Applied to Livestock Production. Editorial Garsi, Madrid, vol VII, 362-367

Walters JR, Sellwood R (1987) Breeding company experience of genetic resistance to disease in pigs. $38^{e}$ Réunion Annuelle, Féd Eur Zootech, Lisbonne, Portugal, 28 septembre- $1^{\text {er }}$ octobre $1987,8 \mathrm{p}$ 